\title{
Age-related differences in evaluating developmental stability
}

\author{
Mustafić, Maida ; Freund, Alexandra M
}

\begin{abstract}
Two studies examined the hypothesis that the evaluation of developmental stability changes across adulthood. Results of Study $1(\mathrm{~N}=119)$ supported the expectation that older adults $(\mathrm{M}$ age $=$ 65.29 years $)$ - compared to younger $(\mathrm{M}$ age $=23.38$ years $)$ and middle-aged adults $(\mathrm{M}$ age $=38.68$ years $)$ evaluate developmental stability more positively and losses less negatively across all life domains included in this study (subjective well-being, social relationships, cognition, physical functioning). Replicating and extending these findings, Study $2(\mathrm{~N}=182$, age-range: $18-86$ years) demonstrated that these age-related differences exist only for stability on an explicit and implicit level of evaluation. Moreover, Study 2 shows that the positive evaluation of stability increases after resource investments into maintaining stability were made salient. We discuss the results in relation to motivational orientation and psychological adjustment to developmental change.
\end{abstract}

DOI: https://doi.org/10.1177/0165025413490866

Posted at the Zurich Open Repository and Archive, University of Zurich ZORA URL: https://doi.org/10.5167/uzh-79370

Journal Article

Originally published at:

Mustafić, Maida; Freund, Alexandra M (2013). Age-related differences in evaluating developmental stability. International Journal of Behavioral Development, 37(4):376-386.

DOI: https://doi.org/10.1177/0165025413490866 


\title{
Age-related differences in evaluating developmental stability
}

\author{
M Mustafic, A M Freund
}




\title{
Running Head: EVALUATING DEVELOPMENTAL STABILITY
}

\author{
Age-Related Differences in Evaluating Developmental Stability \\ Maida Mustafić and Alexandra M. Freund \\ University of Zurich
}

\begin{abstract}
Author Note
Maida Mustafić and Alexandra M. Freund, University of Zurich, Department of Psychology. Maida Mustafić is now at the University of Trier. Correspondence concerning this article may be addressed to either author. Maida Mustafić, University of Trier, Department of Psychology, Educational Psychology, Universitätsring 15, DE-54296 Trier, mustafic@unitrier.de; or Alexandra M. Freund, University of Zurich, Department of Psychology, Applied Psychology: Life Management, Binzmühlestrasse 14/11, CH-8050 Zurich, freund@psychologie.uzh.ch

This research was supported by grants from the Swiss National Science Foundation (Project "Process and outcome focus - The role of age", ID: 100014-116528; PI: Alexandra M. Freund) as well as the Forschungskredit of the University of Zurich (Project "Specificity and Adaptivity of Process and Outcome Goal Focus”, ID: 56230803, awarded to Maida Mustafić). The authors would like to thank the Life-Management team at the University of Zurich for helpful discussions of the work reported in this article and Fabian Lienhard and Thekla Schulze for their assistance during data collection. During the work on this paper, Maida Mustafić was a fellow of the International Max Planck Research School on the Life Course (LIFE).
\end{abstract}




\begin{abstract}
Two studies examined the hypothesis that the evaluation of developmental stability changes across adulthood. Results of Study $1(N=119)$ supported the expectation that older adults $\left(M_{\text {age }}\right.$ $=65.29$ yrs. $)$ - compared to younger $\left(M_{\mathrm{age}}=23.38 \mathrm{yrs}.\right)$ and middle-aged adults $\left(M_{\mathrm{age}}=38.68\right.$ yrs.) - evaluate developmental stability more positively and losses less negatively across all life domains included in this study (subjective well-being, social relationships, cognition, physical functioning). Replicating and extending these findings, Study $2(N=182$, age-range: $18-86$ yrs.) demonstrated that these age-related differences exist only for stability on an explicit and implicit level of evaluation. Moreover, Study 2 shows that the positive evaluation of stability increases after resource investments into maintaining stability were made salient. We discuss the results in relation to motivational orientation and psychological adjustment to developmental change.
\end{abstract}

Keywords: Subjective conceptions of development, motivational orientation, developmental stability, developmental loss, gains, losses, adult development 
Age-Related Differences in Evaluating Developmental Stability

Imagine you just had a thorough physical exam and the result is that your level of physical fitness has not changed since the last exam five years ago. Would you evaluate the stability of your physical fitness as positive or negative? The answer most likely depends on your expectations regarding the development of your physical fitness. If you had expected your

fitness to improve, you most likely evaluate stability negatively. In contrast, if you had expected your fitness to decline, stability might feel like a gain and constitute a positive developmental trajectory. The central hypothesis of the current studies is that the evaluation of developmental stability depends on the gain or loss expectations, respectively. More specifically, as expectations for developmental trajectories reflect increasing losses across adulthood (Heckhausen, Dixon, \& Baltes, 1989; Mustafić \& Freund, 2012), older adults should be more likely than younger or middle-aged adults to evaluate stability as positive.

\section{Age and Expectation of Increasing Losses}

One of the basic tenets of lifespan psychology is that losses increase across adulthood (Baltes, 1997). Increasing losses have been shown empirically in a variety of life domains such as fluid aspects of cognitive functioning, physical health, and physical functioning (Baltes \& Smith, 2003). On the contrary, research shows that the quality of social relationships and subjective well-being remain fairly stable into old age (e.g., Carstensen, Isaacowitz, \& Charles, 1999; Kunzmann, 2008). Importantly, there is high social consensus about the expected losses in older adulthood (Heckhausen et al., 1989). In other words, adults of different ages are aware of the developmental changes (Diehl \& Wahl, 2009). In the following, we argue that adults use expected trajectories as standards of comparison for developmental trajectories.

\section{Evaluation of Stability as a Developmental Outcome}


Subjective outcome expectations determine the evaluation of outcomes of choices as gains or losses and, thereby, the emotional reactions to these outcomes (Mellers, 2000). We apply the same rationale to the evaluation of developmental outcomes. The effect of subjective expectations might be most evident for the evaluation of developmental stability (rather than of clear-cut gains or losses). This should be the case because stability leaves more room for subjective interpretations than marked gains or losses. Older adults might evaluate stability against the expected loss trajectory as a comparison standard, which should lead to a positive evaluation of stability. In contrast, younger and middle-aged adults might evaluate stability against an expected growth trajectory and hence evaluate stability negatively.

Developmental expectations are domain and context specific (Kornadt \& Rothermund, 2011; Mustafić \& Freund, 2012). Thus, we expect the developmental evaluations to differ between different life domains. As older adults expect more losses in the domains of physical and cognitive functioning compared to subjective well-being or social relationships (Study 1, Mustafić \& Freund, 2012), age-related differences in the evaluation of stability should be strongest for the physical and cognitive domain.

\section{Explicit and Implicit Developmental Evaluations}

As has been shown repeatedly for attitudes and evaluations, explicit and implicit judgments do not always converge (for a review see Greenwald \& Banaji, 1995). Gawronski and Strack (2004) proposed that explicit measures reflect an evaluative reaction that might not only be affected by spontaneous affective responses but also reflect the impact of other information that is cognitively available for an evaluation. In contrast, implicit measures capture immediate reactions that are less subject to influences other than those activated by the stimulus (Gawronski, LeBel, \& Peters, 2007; see also the associative-propositional evaluation model, 
Gawronski \& Bodenhausen, 2006). Explicit and implicit processes are both important for regulating developmental losses (e.g., Brandtstädter \& Greve, 1994). With increasing age, losses might threaten positive emotions and subjective health, but only to a degree that they are subjectively appraised as losses (Scherer, 2009). Re-evaluations, such as an increasingly positive evaluation of stability with age, might help to maintain subjective well-being in the face of losses. To function well and efficiently and not to interfere with situational demands, positive evaluations of stability might be most beneficial if they do not only occur explicitly but also implicitly (Koole \& Rothermund, 2011).

\section{The Role of Salience of Resource Investment for Evaluations of Stability}

What other processes might contribute to age-related differences in the evaluation of stability? A developmental outcome might increase in value when it is perceived to be difficult to achieve (Labroo \& Kim, 2009). As people experience a change in resource availability across adulthood, younger and older adults might be differentially sensitive to resource investment (Ebner, Freund, \& Baltes, 2006). Given the decrease in resource availability across adulthood, older adults are more likely than younger adults to be sensitive to how many resources they spend on what (cf. the importance of selectivity in older adulthood; Marsiske, Lang, Baltes, \& Baltes, 1995), and to be highly motivated to maintain their resources and achievements (Freund, 2006). Freund and Riediger (2001) argue that the maintenance of functioning should be particularly important in older adulthood because repairs become more and more costly for older adults (see also Baltes, 1997). Moreover, compared to younger adults, older adults expect declines in functioning in various life domains if they do not invest resources (Mustafić \& Freund, 2012). Therefore, they are more likely to be aware of the necessity to invest resources in order to achieve stability. As people tend to value achievements more when they come at a price 
(such as resource costs; Labroo \& Kim, 2009), older adults should value stability more positively than younger adults. Correspondingly, if the costs of maintaining stability are salient, younger adults should evaluate stability similarly positively as older adults (cf. Ebner et al., 2006).

\section{Present Research and Overview of the Current Studies}

Developmental stability is more open to subjective evaluation than gains and losses. Exploiting this idea, we developed a new method to assess the subjective evaluation of stability. We also assessed whether the evaluation of stability depends on the salience of investment necessary for maintaining stability. Using a newly developed method to operationalize developmental outcomes, the current studies tested age-related differences in evaluations of stability on the explicit as well as the implicit level.

More concretely, the present studies investigated the evaluation of developmental stability as positive or negative depending on age. The first study tested whether the positive evaluation of stability increases with age and differs by life domain (subjective well-being, social relationships, cognition, physical functioning). We expected older adults to evaluate stability more positively in domains in which they expect more severe losses (physical and cognitive functioning) compared to domains that are less associated with losses (social relationships and subjective well-being). As people with high levels of functioning possibly evaluate stability more positively than people with low levels of functioning, we included a measure of current levels of functioning in the first study. The second study tested the hypothesis that age differences in evaluations of stability not only apparent on an explicit but also on an implicit level. Finally, we tested whether the age-related differences in the evaluation of stability disappear when the resources necessary for maintenance of functioning were made salient (Ebner, et al., 2006). 


\section{Study 1}

\section{Method}

\section{Procedure}

After providing informed consent, participants filled out a brief demographic questionnaire. Participants were then introduced to three types of graphic depictions of developmental trajectories (indicating gain, stability, and loss), starting at "now" and ending "in 10 years" in the future (see Appendix A for a depiction of the stimulus material) in four life domains (subjective well-being, social relationships, cognition, physical functioning). Thus, the participants evaluated 12 trajectories in total (three developmental trajectories in four domains). Separately for each domain, they were asked to assess how they would evaluate their own development if it resembled a given trajectory. After the study, participants were fully debriefed. As a way of reimbursing participants, we conducted a lottery of 50 vouchers worth 15 Euro / 20 CHF (approximately 20 USD) for different stores.

\section{Sample}

The total sample was comprised of $N=119$ younger $(n=49 ; 65 \%$ women, $16-29$ years, $\left.M_{\text {age }}=23.28, S D=3.07\right)$, middle-aged $\left(n=29 ; 76 \%\right.$ women; $30-54$ years, $M_{\text {age }}=38.68, S D=$ $1.38)$, and older adults $\left(n=41 ; 56 \%\right.$ women, $57-74$ years, $\left.M_{\text {age }}=65.29, S D=4.7\right)$. Participants were recruited via our participant pool and postings on various Swiss, German, and Austrian websites. Overall, the sample was well educated with $88 \%$ of the younger, $62 \%$ of the middleaged and $49 \%$ of the older adults holding a degree from the highest school track in these countries (Matura).

\section{Measures}


The evaluation of the developmental trajectory was assessed using three items (positive, pleasant, gain) representing a positive evaluation and three items (negative, unpleasant, loss) representing a negative evaluation of the trajectory rated on a scale from 1 (do not agree at all) to 7 (agree completely). The presentation order of the trajectories was randomized. The mean of the three items was treated as a dependent variable measuring the degree of positive evaluation (Cronbach's Alphas: gain $=.76$, stability $=.83$, loss $=.80)$ or negative evaluation $($ gain $=.71$, stability $=.79$, loss $=.77$ ). To test if these evaluations reflected primarily the current levels of functioning, we assessed the current self-reported level of functioning on a scale ranging from $0 \%$ (lowest level of functioning) to $100 \%$ (highest level of functioning) in each domain.

\section{Results and Discussion}

First, there were no significant associations of self-reported aggregated as well as domain specific levels of functioning and age (aggregated: $r=.15, p=.09$; social relationships: $r=.13 p$ $=.15$; physical functioning: $r=.11, p=.25$; subjective well-being: $r=.17, p=.07$; cognition: $r$ $=.11, p=.25,1-\beta=.87 ;$ aggregated mean levels: $M_{\text {younger }}=73.83, S D=14.66 ; M_{\text {middle-aged }}=$ $\left.63.45, S D=24.33 ; M_{\text {older }}=76.47, S D=10.68\right)$.

Hypotheses regarding age and domain differences were tested using a mixed MANOVA with domain and trajectory as within-participant factors and age group as between-participant factor and positive and negative evaluations of the trajectories as the dependent variables. Analyses of repeated measures ANOVA reveal the same results and supplemental analyses controlling for gender and education did not change the results.

The 4 (domain: subjective well-being, social relationships, cognition, physiological functioning) x 3 (trajectory: gain, stability, loss) x 3 (age group: young, middle-aged, older) MANOVA revealed a significant effect of age on the combined dependent variables $(F(4,230)=$ 
$\left.7.53, p<.001, \eta^{2}=.12\right)$ and a main effect of trajectory $\left(F(4,113)=294.10, p<.001, \eta^{2}=.91\right)$.

The domain effect was not significant $\left(F(6,111)=1.26, p=.24,1-\beta=.60, \eta^{2}=.06\right)$. As expected, the two-way interaction of trajectory $\mathrm{x}$ age group was significant $(F(8,226)=6.94, p<$ $\left..001, \eta^{2}=.20\right)$, but not the interaction of domain $\mathrm{x}$ age group $(F(12,222)=1.3, p=.24,1-\beta=$ $\left..71, \eta^{2}=.06\right)$; and, contrary to expectations, neither was the three-way interaction of age group $\mathrm{x}$ trajectory $\mathrm{x}$ domain $\left(F(24,210)=1.26, p=.28,1-\beta=.87, \eta^{2}=.11\right)$.

\section{Age-Differences in the Evaluations of Trajectories}

Following up the trajectory $\mathrm{x}$ age group interaction, we ran univariate ANOVAs with age group as a between-participants factor and positive and negative evaluations of the trajectories as dependent variables. The results are displayed in Figure 1.

There was no difference between the age groups in the evaluation of gain trajectories (positive evaluations: $F(2,116)=.551, p \geq .58,1-\beta=.14$; negative evaluations: $F(2,116)=$ $1.09, p \geq .34,1-\beta=.24)$. As predicted, significant age-group differences emerged in the evaluation of the stability trajectories (positive evaluation: $F(2,116)=21.96, p<.001, \eta^{2}=.14$; negative evaluation: $\left.F(2,116)=1.09, p \geq .34, \eta^{2}=.17\right)$. Confirming expectations, follow-up comparisons (Scheffé-Tests) showed that older adults evaluated the stability trajectories more positively $(M=5.62, S D=1.48)$ and less negatively $(M=2.14, S D=.93)$ than younger (positive: $M=4.11, S D=1.51, d=1.02$; negative: $M=3.23, S D=1.37, d=.93$ ) and middleaged adults (positive: $M=3.94, S D=1.26, d=1.22$; negative: $M=3.35, S D=1.41, d=.98$; both $p s \leq .001)$. Younger and middle-aged adults did not differ in the positive and negative evaluations of the stability trajectories (positive: $d=.12$, negative: $d=.09 ; p \geq .84$ ).

There was no significant difference between the age groups regarding the positive evaluation of loss trajectories $(F(2,116)=2.57, p \geq .08,1-\beta=.50)$. However, age groups 
differed in how negatively they evaluated loss trajectories $\left(F(2,116)=7.96, p<.001, \eta^{2}=.12\right)$.

Older adults evaluated loss trajectories less negatively $(M=5.42, S D=1.39)$ than younger $(M=$ $6.33, S D=.93 ; d=.79)$ and middle-aged adults $(M=6.15, S D=1.09, d=.58$; both $p s<.05)$.

Younger and middle-aged adults did not differ from each other in the negative evaluations of the loss trajectories $(d=.18, p \geq .96)$.

Finally, multiple regression analyses were used to predict the positive and negative evaluations of stability from current levels of functioning and age. As expected, persons with high current levels of functioning evaluated stability more positively and less negatively $\left(\beta_{\text {positive }}\right.$ $\left.=.60, t(111)=8.17, p<.001 ; \beta_{\text {negative }}=-.53, t(117)=-6.71, p<.001\right)$. Importantly, there was no interaction of age and current levels of functioning in predicting the evaluations $\left(\beta_{\text {positive }}=\right.$ $\left..10, t(115)=1.53 ; p=.13 ; \beta_{\text {negative }}=-.03, t(115)=-.34, p=.73\right)$.

Taken together, results indicate that there was consensus across age groups regarding the subjective evaluation of clear-cut gain and loss trajectories: Gains are positive and losses are negative - regardless of age. However, age-related differences in the evaluation of developmental trajectories emerged in the evaluation of stability. As predicted, older adults evaluated stability trajectories more positively and less negatively than younger and middle-aged adults. Furthermore, we found an "evaluation split" regarding the loss trajectories. There was agreement that losses are not positive. However, older adults evaluated the loss trajectory as less negative than younger and middle-aged adults.

\section{Study 2}

To replicate and generalize results of Study 1, we examined evaluations using a different assessment method and another sample. More concretely, Study 2 was aimed at assessing evaluations on an explicit level (similar to the assessment in Study 1) as well as on an implicit 
level (using the affective misattribution paradigm by Payne, Cheng, Govorun, \& Stewart, 2005). Further, we examined age-related differences in the sensitivity to resource investment when evaluating stability. In order to test the context dependency of explicit evaluations, we again used trajectories in four functional life domains (subjective well-being, social relationships, cognition, physical functioning).

\section{Method}

\section{Procedure}

After providing informed consent, participants were introduced to graphic illustration of developmental trajectories. Developmental trajectories were graphically depicted in form of an ascending line (gain), a horizontal line (stability), or a descending line (loss) in an axis of abscissas with the abscissa denoting age (younger, middle-aged, older adulthood) and the ordinate the of level of functioning (ranging from $0 \%$ to $100 \%$ ). After having been introduced to these graphs, participants were asked to draw their personal trajectory in the four functional domains (subjective well-being, social relationships, cognition, physical functioning). They were encouraged to ask clarification questions. This procedure has already been successfully implemented in previous studies (Mustafić \& Freund, 2012) showing that adults easily understand and draw graphical depictions of development (upward lines symbolizing gains, downward lines symbolizing losses and horizontal lines symbolizing stability).

Explicit evaluations. To assess explicit evaluations, participants were seated in front of a computer and told that a slide will be presented on the computer screen announcing the functional domain to which the subsequent trajectory should be applied. Participants were told that the second picture will show the graphical depiction of a developmental trajectory (gain, stability, or loss) illustrated in an axis of abscissa with time ranging from "now" to "in 10 years" 
and the ordinate depicting the level of functioning ( $0 \%-100 \%$; see Appendix B). Following this picture, a pattern mask consisting of black and white "noise" appeared. This was done in order to keep the procedure of the explicit and the implicit assessment (described below) as similar as possible and thereby ensure their comparability. Participants were asked to "spontaneously evaluate" the trajectory using a key labeled pleasant or unpleasant when the pattern mask appeared. The mask disappeared as soon as the participant responded. Pilot testing with two younger and three older participants revealed that presentation times of $300 \mathrm{~ms}$ for the presentation of the functional domain and for the trajectory graph are adequate for all three age groups studied. The task lasted approximately 5 minutes. Participants completed 36 randomly ordered trials.

Implicit evaluations. Implicit evaluations were assessed using the affect misattribution paradigm (AMP, Payne et al., 2005). This task was again presented on the computer, and, similarly to the explicit measure, participants were told that they were going to see a graph representing a developmental trajectory (gain, stability, loss) using the identical stimuli as in the explicit evaluation task. The first screen was followed by a Chinese pictograph. Following Payne et al., the trajectory and the Chinese pictograph were presented for $250 \mathrm{~ms}$. After the pictograph, a pattern mask consisting of black and white "noise" appeared until participants responded if they evaluated the pictograph as pleasant or unpleasant. The AMP is regarded as an implicit measure of evaluation as the pleasant or unpleasant reaction elicited by the trajectory is carried over and implicitly "misattributed" to the pictograph. It is robust even when participants are instructed to intentionally counteract a potential carry-over effect (Payne et al. 2005). In total, the task lasted approximately 5 minutes. Each trajectory was presented four times following 12 Chinese pictographs, resulting in 48 trials. Developmental trajectories were 
randomized, as were the functional domains (domains were included only in the explicit measure). The implicit measure did not differentiate trajectories by domain, as the misattribution should only reflect the affective reaction to the trajectory and not confound the affective reaction to the trajectory and the affective reaction to the functional domain.

Explicit evaluations after manipulation of resource investment salience. In the last part of the study, we varied the salience of resource investment. For this purpose, we randomly assigned participants to one of two salience conditions (high salience vs. control condition), resulting in a between-participant design for this part of the study. The control group was asked to evaluate stability in the four life domains in the same way as in Study 1 (i.e., no mentioning of resource investment). The resource-salience group was instructed to evaluate stability after reading instructions that described the necessity of resource investment in order to maintain functioning over time (e.g., to "regularly take care of friends" in order to achieve stability in the domain of social relationships or to "exercise regularly" in order to maintain physical functioning, see Appendix $\mathrm{C}$ for an example). The first set of explicit and implicit evaluations was assessed using EPrime (Version 2.0); the third set of evaluations depending on the salience of resource investment needed to maintain stability was collected online with the survey program www.soscisurvey.de. The presentation of all stimuli within one condition was randomized. The experiment lasted approximately 30 minutes in total and was reimbursed with $10 \mathrm{CHF}$ (approximately 11.30 USD). At the end of the study, participants were thanked and fully debriefed.

\section{Sample}

Participants were recruited via the participant pool of our laboratory or approached directly 
on the university campus. The total sample was comprised of $n=60$ younger adults ( $48 \%$ women, 18 -35 years, $\left.M_{\mathrm{age}}=25.38, S D=4.09\right), n=54$ middle-aged adults ( $63 \%$ women; $38-59$ years, $\left.M_{\text {age }}=48.55, S D=5.85\right)$, and $n=68$ older adults ( $57 \%$ women, $60-84$ years, $M_{\text {age }}=71.70$, $S D=5.18) .95 \%$ of the younger, $55 \%$ of the middle-aged and $35 \%$ of the older adults held a degree of the highest school track in Switzerland (Matura).

\section{Measures}

Explicit and implicit evaluations of trajectories. Following Payne et al. (2005), to determine the explicit and implicit evaluations of the gain, stability, and loss trajectories, we calculated an explicit and implicit ratio of "pleasant" to total responses for each trajectory and treated these ratios as dependent variables in our analyses. Whereas the explicit and implicit ratios for the gain trajectory were not correlated, the explicit and implicit ratios of stability and loss trajectories were positively correlated (gain: $r=-.08, p=.82$; stability: $r=.25, p<.001$; loss: $r=.20, p=.05)$. The evaluation of the stability trajectory after the manipulation of perceived investment was assessed using the same procedure as in Study 1.

\section{Results}

\section{Explicit Evaluations of Trajectories}

Results regarding explicit and implicit evaluations of trajectories are depicted in Figure 2. Hypotheses regarding explicit evaluations were tested using a mixed MANOVA with domain as a within-participants factor, age group as between-participant factor, and the ratio of positive explicit responses to the gain, stability, and loss trajectories as dependent variables. Analyses of repeated measures ANOVA reveal the same results and supplemental analyses controlling for gender and education did not change the results. 
The 4 (domain: subjective well-being, social relationships, cognition, physiological functioning) x 3 (age group: young, middle-aged, older) MANOVA revealed a significant effect of age group $\left(F(6,304)=9.07, p<.001, \eta^{2}=.15\right)$. Neither the effect of domain $(F(9,146)=$ $\left.1.85, p=.06,1-\beta=.80, \eta^{2}=.73\right)$, nor the interaction of domain $\mathrm{x}$ age group $(F(18,292)=.83$, $\left.p=.66,1-\beta=.61, \eta^{2}=.05\right)$ was significant.

Follow-up comparisons between age groups showed that there were significant differences between age groups in the evaluation of all three trajectories (gain: $F(2,179)=3.21$, $p<.05, \eta^{2}=.03$; stability: $F(2,179)=5.00, p<.05, \eta^{2}=.05$; loss: $F(2,179)=27.57, p<.001, \eta^{2}$ $=.23$ ). Post-hoc Scheffé-Tests revealed that younger adults evaluated the gain trajectories significantly more positively $\left(M_{\text {young }}=.94, S D=.15\right)$ than older adults $\left(M_{\text {older }}=.85, S D=.22, d\right.$ $=.47, p=.05)$. Younger and middle-aged adults $\left(M_{\text {middle-aged }}=.87, S D=.21, d=.39, p=.22\right)$ as well as older and middle-aged adults did not differ regarding the evaluation of gain trajectories $(d=.09, p=.84)$. Replicating results of Study 1 , older adults evaluated the stability trajectories $\left(M_{\text {older }}=.74, S D=.30\right)$ more positively than younger adults $\left(M_{\text {young }}=.56, S D=.36, d=.55, p=\right.$ $.01)$. Older and middle-aged ( $\left.M_{\text {midddle-aged }}=.69, S D=.33, d=.55, p=.68\right)$ as well as younger and middle-aged adults $(d=.38, p=.12)$ did not differ. Finally, regarding loss trajectories, older adults provided a more positive evaluation $\left(M_{\text {older }}=.45, S D=.34\right)$ than middle-aged ( $M_{\text {middle-aged }}$ $=.28, S D=.31, d=.52)$ and younger adults $\left(M_{\text {young }}=.07, S D=.18, d=1.38\right.$, both $p$ s $\left.<.006\right)$. Middle-aged adults evaluated the loss trajectories more positively than younger adults $(d=.85, p$ $=.001)$.

\section{Implicit Evaluations of Trajectories}

Hypotheses regarding implicit evaluations were tested using a MANOVA with age group as between-participant factor, and the ratio of positive implicit responses to the Chinese 
characters following gain, stability, and loss trajectories as dependent variables. The MANOVA evinced a significant main effect of age group $\left(F(6,360)=3.60, p=.002, \eta^{2}=.06\right)$ : Older adults generally evaluated Chinese characters $\left(M_{\text {older }}=.68, S D=.20\right)$ more positively than younger $\left(M_{\text {young }}=.54, S D=.20, d=71\right)$ and middle-aged adults $\left(M_{\text {middle-aged }}=.57, S D=.18, d=.58\right.$; both $p \mathrm{~s}<.05, d=58)$. Younger and middle-aged adults did not differ $(d=.16, p=.72)$. Post-hoc comparisons using Scheffé-Tests (see Figure 2) revealed that older adults evaluated the Chinese character following a gain trajectory significantly more positively than younger adults $\left(M_{\text {older }}=\right.$ $.74, S D=.22 ; M_{\text {young }}=.60, S D=.27, d=.58 ; p=.006$ ); older and middle-aged adults did not $\operatorname{differ}\left(M_{\text {middle-aged }}=.67, S D=.24, d=.31, p=.31\right)$; middle-aged and younger adults did not differ $(d=.28 ; p=.30)$. Similarly, older adults evaluated the Chinese character after the stability trajectory more positively than younger $\left(M_{\text {older }}=.73, S D=.26 ; M_{\text {young }}=.55, S D=.24, d=.72 ; p\right.$ $<.001)$ and middle-aged adults $\left(M_{\text {middle-aged }}=.60, S D=.25, d=.51 ; p=.01\right)$; again, middle-aged and younger adults did not differ $(d=.21 ; p=.7)$. Finally, older adults evaluated the Chinese character following a loss trajectory significantly more positively than middle-aged adults $\left(M_{\text {older }}\right.$ $=.56, S D=.30 ; M_{\text {middle-aged }}=.46, S D=.27 d=.35 ; p=.05$ ); older and younger adults did not differ, $\left(M_{\text {young }}=.45, S D=.27, d=.39 ; p=.11\right)$, neither did younger and middle-aged adults $(d=$ $.04, p=.92)$.

\section{Salience of Resource Investment}

The importance of the salience of resource investment for positive evaluations of stability was investigated using a mixed MANOVA with condition and age group as between-participant factors, domain as within-participant factor and positive and negative evaluations of stability as dependent variables. The 2 (condition: no resource manipulation, resource manipulation) x 3 (age group: young, middle-aged, older) x 4 (domain: subjective well-being, social relationships, 
cognition, physical functioning) mixed MANOVA evinced a significant effect of age group $(F(4$, $\left.348)=9.75, p<.001, \eta^{2}=.08\right)$, and a significant main effect of condition $(F(2,174)=7.39, p<$ $\left..001, \eta^{2}=.10\right)$. Neither the main effect of domain $\left(F(6,170)=1.42, p=.21,1-\beta=.54, \eta^{2}=\right.$ .05 ) nor any of the two- and three-way interaction effects were significant (age group $\mathrm{x}$ condition: $F(4,348)=1.33, p=.261,1-\beta=.41, \eta^{2}=.01$; domain $\mathrm{x}$ condition $F(6,170)=.70, p$ $=.65,1-\beta=.27, \eta^{2}=.02$; domain $\mathrm{x}$ age group: $\left.F(12,340)=1.54, p=.11,1-\beta=.82, \eta^{2}=.04\right)$; domain $\mathrm{x}$ condition $\mathrm{x}$ age group: $\left.F(12,340)=1.18, p=.29,1-\beta=.68, \eta^{2}=.04\right)$.

Follow-up analyses (Scheffé-Tests) of the main effect of age group revealed that older adults evaluated the stability trajectory less negatively $\left(M_{\text {older }}=2.27, S D=1.31\right)$ and more positively $\left(M_{\text {older }}=5.81, S D=1.29\right)$ than younger adults (negative: $M_{\text {young }}=2.83, S D=1.05, d=$ .48; positive: $\left.M_{\text {young }}=4.68, S D=1.04, d=.98, p \mathrm{~s}<.05\right)$. Younger and middle-aged adults (negative: $M_{\text {middle-aged }}=2.29, S D=1.34$, positive: $M_{\text {middle-aged }}=5.33, S D=1.44$ ) differed in the positivity of the evaluation of gain trajectories $(d=.98, p<.05)$, but not in the negativity ( $d=$ $.45, p \leq .76$ ). Older and middle-aged adults did not differ (positive: $d=.47, p=.12$; negative: $d$ $=.36, p=.99)$.

Indicating a successful manipulation, across age groups participants evaluated stability more positively $\left(F(2,175)=16.51, p<.001, \eta^{2}=.16\right)$ and less negatively $(F(2,175)=4.85, p=$ $.009, \eta^{2}=.05$ ) in the resource salience condition compared to the control. Similarly, comparing the explicit evaluations at the beginning of the experiment with the evaluations at the end of the experiment within the resource manipulation group, shows that the positive explicit evaluation of stability increased after the manipulation (effect of condition: $F(1,83)=5.59, p=.02, \eta^{2}=.06$ ).

Most importantly and confirming our hypotheses, the second study replicated results of Study 1 regarding age-related differences in the explicit and implicit evaluation of stability. 
Regarding the evaluation of gain and loss trajectories, implicit evaluations did not conform to the explicit pattern of age-related differences. Further, results suggest that the salience of resource investments contributes to a positive evaluation of stability. Contrary to our expectations, however, the resource manipulation did not eliminate the age-group differences.

\section{General Discussion}

Returning to our opening example - whether individuals will react positively or negatively to the news that our physical fitness has stayed stable over the past five years is likely to depend on whether individuals had expected an increase or a decrease in physical functioning. As expectations of personal functioning reflect increasing losses across adulthood (Heckhausen, Dixon, \& Baltes, 1989, Mustafić \& Freund, 2012), we hypothesized that older adults evaluate stability more positively than younger adults. This is what the results of the current two studies suggest. More specifically, the affective evaluation of stability changes across adulthood such that with increasing age, stability becomes explicitly and implicitly more positive whereas losses become less negative. Regarding middle adulthood, the results of both studies suggest that middle-aged adults evaluate stability more positively than younger, but still less positively than older adults. This was expected as middle-aged adults still acquire gains (e.g., knowledge) but they are also confronted with initial losses (e.g., death of parents or losses of visual acuity). On a methodological level, the studies present new methods to investigate subjective evaluations of stability.

\section{The Role of Resources in Evaluations of Stability}

One of the factors contributing to age-related differences in the evaluation of stability trajectories might be that it is more resource intense to achieve stability in older compared to younger or middle adulthood. Moreover, previous research on goal orientation suggested that 
with increasing age, as resources become scarcer, older adults become more sensitive to resource restrictions (Ebner et al., 2006). As people tend to value achievements and things more when their costs are higher (Labroo \& Kim, 2009), older adults were expected to view stability as more positive than younger or middle-aged adults. Following this rationale, we included an experimental condition that emphasized the resource costs involved in maintaining functioning (i.e., stability). In line with our hypothesis, reminding participants of the resource investment necessary to maintain stability induced more positive evaluations of developmental stability, but did not eliminate age differences in evaluations of developmental stability. However, the main effect of the resource investment manipulation indicated that, in addition to a changing comparison standard, the salience of resource investments seems to contribute to how positively stability is evaluated. To completely reverse younger and middle-aged adults' evaluation of stability, however, the manipulation in the current study might have been too subtle. It might be that people need to experience losses in resources in order to esteem stability. In addition, the samples of the two studies were fairly resource rich as indicated by the high level of education and functional levels. Younger and middle-aged adults who have experienced severe limitations of resources might be more sensitive to such experimental manipulations of resource investment necessary for maintaining functioning.

What might be other explanations why people's positive evaluation of stability increases across adulthood? Inglehart and Baker (2000) suggested that values change according to a scarcity of options. According to this hypothesis, individuals assess the attainability of options with respect to themselves and others. The scarcity estimation of positive outcomes might become more pronounced in older adults because, for example, older adults evaluate selfdevelopment and the development of their age group as less advantageous than younger adults 
(Heckhausen \& Brim, 1997). Stability might be assessed as a rare event for older adults and, therefore, be evaluated more positively. Further studies that induce or assess the perceived attainability of stability might help advance the test for the processes in changing evaluations. It cannot be ruled out that the suggested associations might also be reversed, with people evaluating stability more positively and therefore perceiving stability as scarcer and more valuable with increasing age. Experimental studies would be desirable to test the strength of the two explanation directions.

\section{Lack of Domain Differences}

Previous research suggests that subjective conceptualizations of development are multidimensional (Mustafić \& Freund, 2012). Interestingly, the current studies suggest that evaluations of developmental trajectories are unidimensional. There were no differences in the evaluation of stability between the domains of subjective well-being, social relationships, cognition, and physical functioning. To evaluate developmental gains, stability, and losses, the direction of comparison standard (gains or losses) seemed to be more relevant than the degree of gain and loss expectations within specific life domains. The lack of domain differences suggests that there is a generalized negative evaluation of stability in younger adulthood and an equally generalized positive evaluation of stability in middle and older adulthood across various domains of functioning. However, the current study only included four domains of functioning and more research is needed covering a more diverse area of life domains (e.g., separating cognitive functioning in fluid and crystallized components, social relationships in quantity and quality) as a more stringent test of multidimensionality in the evaluation of developmental trajectories.

\section{Divergent Explicit and Implicit Measures}


Age-patterns for explicit and implicit evaluations of gain and loss trajectories diverge. Explicitly, there were no age-related differences in the evaluations of gains as positive and losses as negative. On the implicit level, older adults evaluated gain, stability, and loss trajectories more positively than younger and middle-aged adults. One of the reasons for the divergent results of explicit and implicit measures of gain and loss trajectories might be methodological and concerns the possibility of a differential structural fit of research designs for explicit and implicit measures (e.g., difference in presentation times of the stimuli, comparing explicit measures assessing evaluations using Likert scales with implicit measures using dichotomous scales). This is rather unlikely, however, as the research design in Study 2 included the same procedure and measures that were used in Study 1.

There are several other explanations for the divergence. Factors such as evaluation strength, evaluation importance, dimensionality of the evaluation, self-presentation concerns, and the magnitude of the discrepancy of the evaluation against a perceived norm might impact whether participants show the same implicit and explicit response (Nosek, 2005). Following these assumptions, there might be a social norm to evaluate a gain trajectory positively and a loss trajectory negatively explicitly and this norm is only considered when people evaluate trajectories explicitly. Finally, the trajectories also might have activated semantic associations related to the trajectories that might have driven the implicit effect (i.e., gain trajectories evoking "gain” and loss trajectories evoking "loss” semantics; Blaison, Imhoff, Hühnel, Hess \& Banse, 2012). Thus, it might be possible to evoke age differences only using decontextualized lines or using words. Further studies using alternative implicit measures are needed to better understand the dissociation of explicit and implicit evaluations of developmental gain and loss trajectories.

\section{Shortcomings}


One of the shortcomings of the current studies is that they involved cross-sectional comparisons between age groups. As is always true for cross-sectional designs, the current research cannot disentangle age and cohort effects (Baltes, 1968). Additionally, in the current studies, the depiction of the trajectories was held rather simple with the three trajectories of linear increase, linear decrease, or stability. Typically, development occurs not perfectly linear and happens in concert with changes in different domains. However, as a first step, the use of linear trajectories was the most parsimonious way to investigate differences in evaluations and could also be used for the implicit assessment. Future research might broaden the findings on evaluations using more multivariate or asymptotic patterns of developmental trajectories.

Furthermore, future studies are needed to test if people do, in fact, use their personal developmental expectations regarding their own development as a standard of comparison to evaluate developmental trajectories. Previous research suggests that older adults - when explicitly confronted with stereotypic information - use negative expectations for "most others" as a contrasting comparison standard for their own development (Heckhausen \& Krueger, 1993).

Additionally, the order of the tasks was held constant across participants so that the explicit task might have prepared and strengthened a similar reaction in the implicit task. As the implicit and explicit results reveal different patterns, the order of the presented material (explicit and then implicit) seems not problematic. If the study had revealed the same results using the explicit and implicit methods, either a randomized presentation or a factor controlling for presentation order within the design would have been necessary. Ideally, the second part of Study 2 that tested stability evaluation depending on resource investment salience should have been an independent sample instead of collecting data on participants who were already engaged in two related tasks. 


\section{Implications and Outlook}

The research presented here aimed at demonstrating age-related differences in the evaluation of developmental trajectories of gain, stability, and decline using explicit and implicit measures. Taking a constructivistic perspective, we assumed that when people are asked to evaluate stability as a developmental outcome, they would evaluate stability positively or negatively depending on their age. Results support the assumption that the evaluations of stability become more positive with age. Following Brandtstädter's (2006) model of assimilation and accommodation, the adjustment of aspiration levels, such as the positive evaluations of stability, might reflect an accommodation process across the lifespan. Accommodation processes are theorized to reflect an internal flexible adjustment to changing contextual demands. In response to losses, successful self-regulation mechanisms, such as reevaluations, can buffer the negative effects of losses on subjective well-being (Rothermund \& Brandtstädter, 2003).

This research might also serve as a starting point for future studies including more differentiated emotional reactions going beyond positive and negative evaluations of development and more differentiated comparisons. For instance, older adults might experience relief when a loss they expected does not occur. Younger adults might be disappointed if they gain less than they thought they might. Thus, we hope that the current studies contribute to the growing research on subjective developmental conceptualizations by starting to address the important question of the evaluation of developmental trajectories. 


\section{References}

Baltes, P. B. (1968). Longitudinal and cross-sectional sequences in the study of age and generation effects. Human Development, 11, 145-171. doi: 10.1159/000270604

Baltes, P. B. (1997). On the incomplete architecture of human ontogeny: Selection, optimization, and compensation as foundation of developmental theory. American Psychologist, 52, 366-380. doi: 10.1037/0003-066X.52.4.366

Baltes, P. B., \& Smith, J. (2003). New frontiers in the future of aging: From successful aging of the young old to the dilemmas of the fourth age. Gerontology, 49, 123-135. doi: $10.1159 / 000067946$

Blaison, C. Imhoff, R., Hühnel. I., Hess, U., \& Banse, R. (2012). The affect misattribution procedure: Hot or not? Emotion, 12(2), 403-412. doi: 10.1037/a0026907

Brandtstädter, J. (2006). Adaptive resources in later life: Tenacious goal pursuit and flexible goal adjustment. In M. Csikszentmihalyi \& I. S . Csikszentmihalyi (Eds.), A life worth living: Contributions to positive psychology (pp. 143-164). New York: Oxford University Press.

Brandtstädter, J. \& Greve, W. (1994). The aging self: Stabilizing and protective processes. Developmental Review, 14, 52-80.

Carstensen, L. L., Isaacowitz, D. M., \& Charles, S. T. (1999). Taking time seriously: A theory of socioemotional selectivity. American Psychologist, 54, 165-181. doi: 10.1037/0003066X.54.3.165

Diehl, M. K., \& Wahl, H. W. (2009). Awareness of Age-Related Change: Examination of a (Mostly) Unexplored Concept. The Journals of Gerontology Series B: Psychological Sciences and Social Sciences, 65B(3), 340-350. doi:10.1093/geronb/gbp110 
Ebner, N. C., Freund, A. M., \& Baltes, P. B. (2006). Developmental changes in personal goal orientation from young to late adulthood: From striving for gains to maintenance and prevention of losses. Psychology and Aging, 21, 664-78. doi: 10.1037/08827974.21.4.664

Freund, A. M., \& Riediger, M. (2001). What I have and what I do: The role of resource loss and gain throughout life. Applied Psychology: An International Review, 50(3), 370-380. doi: 10.1111/1464-0597.00063

Gawronski, B., \& Bodenhausen, G. V. (2006). Associative and propositional processes in evaluation: An integrative review of implicit and explicit attitude change. Psychological Bulletin, 132, 692-731. doi: 10.1037/0033-2909.132.5.692

Gawronski, B., LeBel, E. P., \& Peters, K. R. (2007). What do implicit measures tell us? Scrutinizing the validity of three common assumptions. Perspectives on Psychological Science, 2, 181-193. doi: 10.1111/j.1745-6916.2007.00036.x

Gawronski, B., \& Strack, F. (2004). On the propositional nature of cognitive consistency: Dissonance changes explicit, but not implicit attitudes. Journal of Experimental Psychology, 40, 535-542. doi: 10.1016/j.jesp.2003.10.005

Greenwald, A. G., \& Banaji, M. R. (1995). Implicit social cognition: Attitudes, self-esteem, and stereotypes. Psychological Review, 102, 4-27. doi: 10.1037/0033-295X.102.1.4

Heckhausen, J., \& Brim, O. G. (1997). Perceived problems for self and other: Self-protection by social downgrading throughout adulthood. Psychology and Aging, 12, 610-619. doi: $10.1037 / 0882-7974.12 .4 .610$

Heckhausen, J., Dixon, R. A., \& Baltes, P. B. (1989). Gains and losses in development throughout adulthood as perceived by different adult age groups. Developmental 
Psychology, 25(1), 109-121. doi: 10.1037/0012-1649.25.1.109

Heckhausen, J., \& Krueger, J. (1993). Developmental expectations for the self and most other people: Age grading in three functions of social comparison. Developmental Psychology, 29(3), 539-548. doi: 10.1037/0012-1649.29.3.539

Inglehart, R., \& Baker, W. E. (2000). Modernization, cultural change, and the persistence of traditional values. American Sociological Review, 65(1), 19-51. doi:10.2307/2657288

Koole, S. L., \& Rothermund, K. (2011). "I feel better but I don't know why": The psychology of implicit emotion regulation. Cognition and Emotion, 25, 389-399. doi:

$10.1080 / 02699931.2010 .550505$

Kornadt, A. E., \& Rothermund, K. (2011). Internalization of age stereotypes into the selfconcept via future self-views: A general model and domain-specific differences. Psychology and Aging. doi: 10.1037/a0025110

Kunzmann, U. (2008). Differential age trajectories of positive and negative affect: Further evidence from the Berlin Aging Study. The Journals of Gerontology, Series B: Psychological Sciences, 63B, 261-270. doi: 10.1093/geronb/63.5.P261

Labroo, A. A. \& Kim, S (2009). The "Instrumentality" Heuristic. Psychological Science, 2009, 20(1), pp. 127-34. doi: 0.1111/j.1467-9280.2008.02264.x

Marsiske, M., Lang, F. B., Baltes, P. B., \& Baltes, M. M. (1995). Selective optimization with compensation: Life-span perspectives on successful human development. In R. A. Dixon \& L. Baeckman (Eds.), Compensating for psychological deficits and declines: Managing losses and promoting gains (pp. 35-79). Mahwah, NJ: Erlbaum. 
Mellers, B. A. (2000). Choice and the relative pleasure of consequences. Psychological Bulletin, 126, 910-924. doi: 10.1037/0033-2909.126.6.910

Mustafić, M., \& Freund, A. M. (2012). Multidimensionality in developmental conceptions across adulthood. Journal of Gerontopsychology and Geriatric Psychiatry, 25, 57-72. doi: $10.1024 / 1662-9647 / \mathrm{a} 000055$

Payne, B. K., Cheng, C. M., Govorun, O., \& Stewart, B. D. (2005). An inkblot for attitudes: Affect misattribution as implicit measurement. Journal of Personality and Social Psychology, 89(3), 277-293. doi: 10.1037/0022-3514.89.3.277

Rothermund, K., \& Brandtstädter, J. (2003). Age stereotypes, self-views, and well-being in later life: Evaluating rival assumptions. International Journal of Behavioral Development, 27, 549-554. doi: 10.1080/01650250344000208 


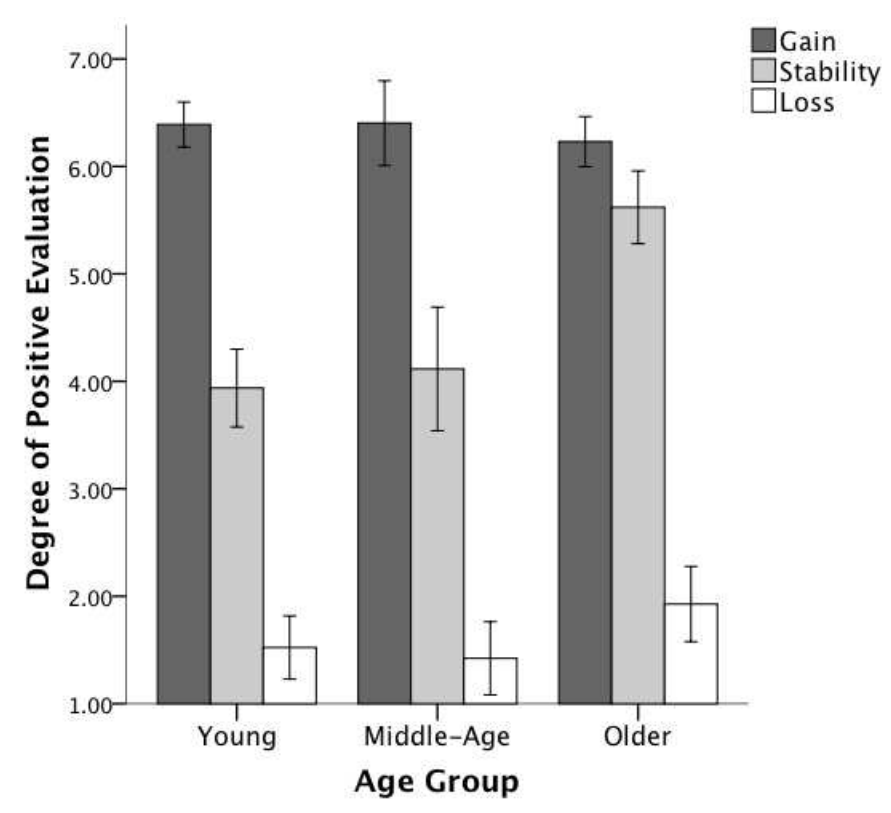

A

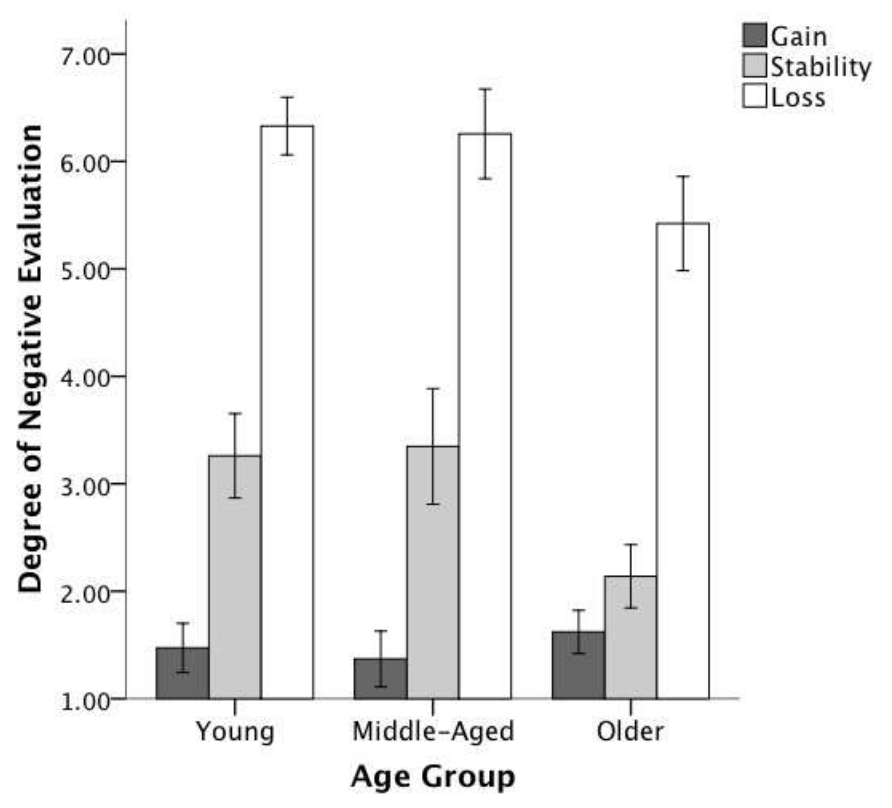

B

Figure 1. Mean (A) positive or (B) negative evaluations of the gain, stability, and loss trajectory across four life domains by age group. The positive evaluation of stability increases and the negative evaluation decreases across adulthood. Error bars represent 95\% confidence intervals. 


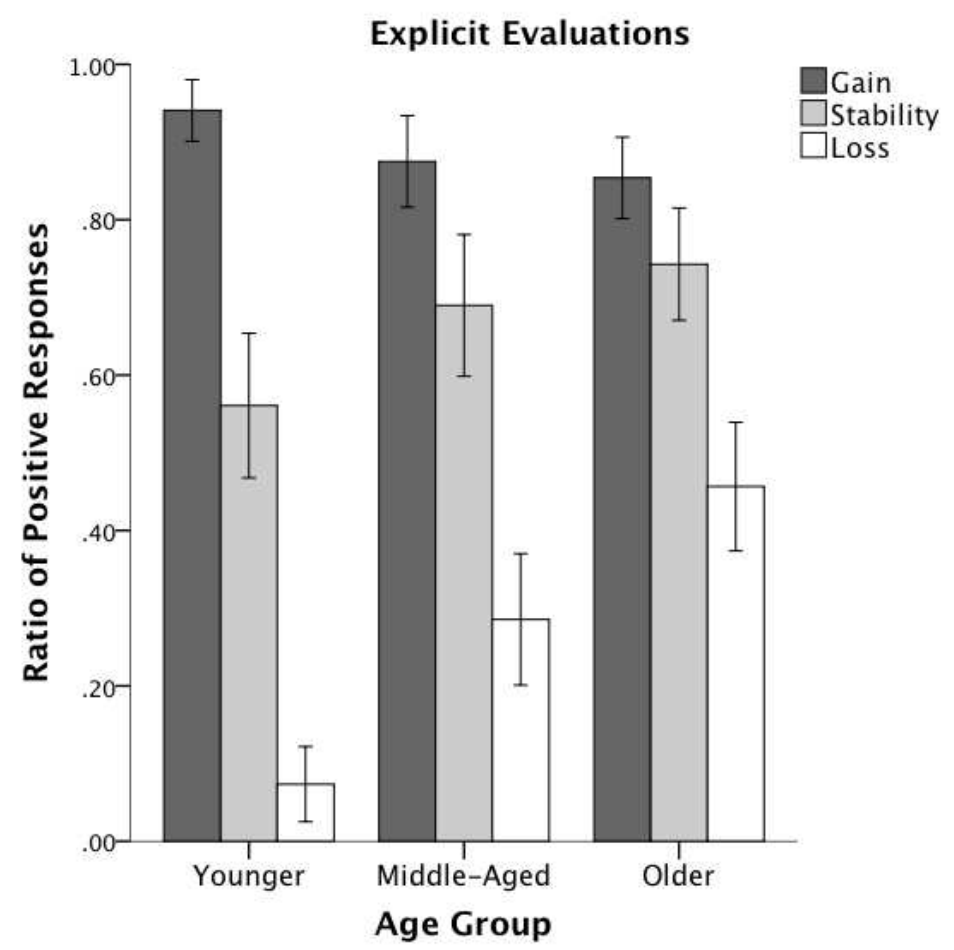

B

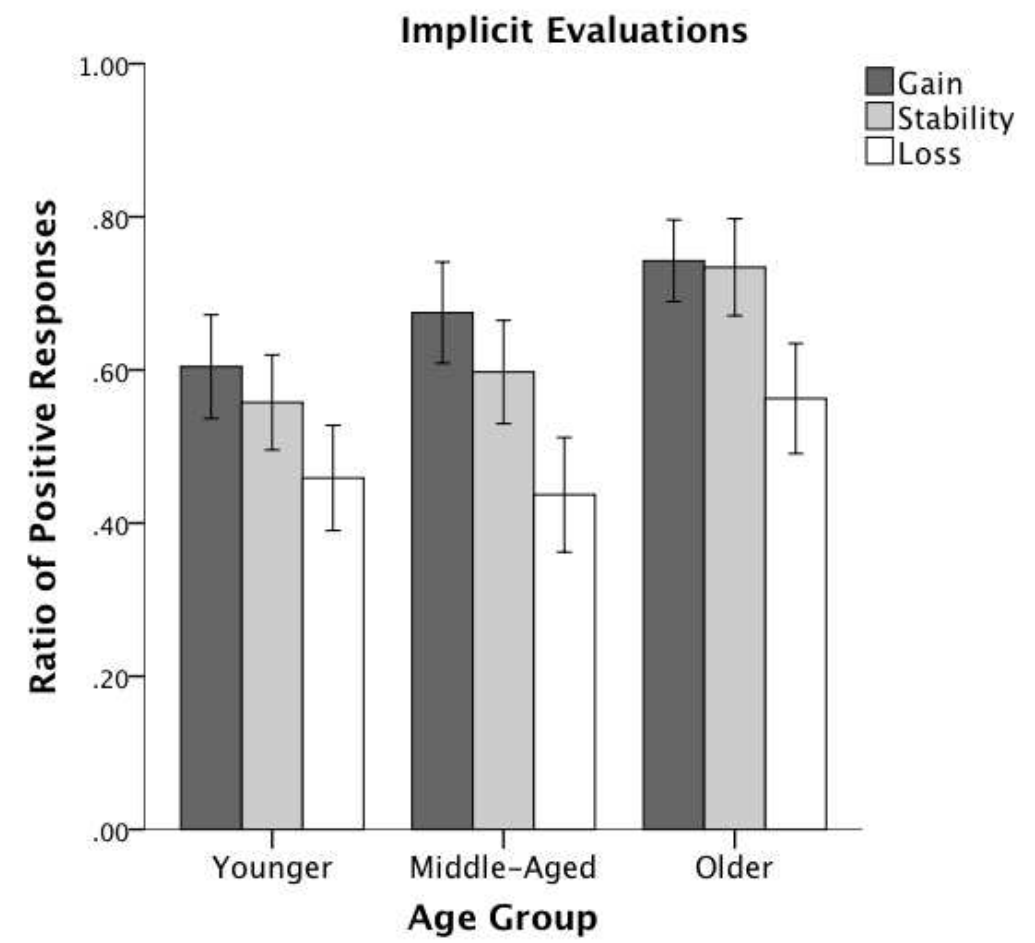

Figure 2. Mean ratio of positive to total responses to gain, stability, and loss trajectory evaluations measured (A) explicitly and (B) implicitly by age group. The positive evaluation of stability increases explicitly and implicitly. Error bars represent confidence intervals. 


\section{Appendix A}

\section{Stimulus Material Used in Study 1 to Assess the Evaluation of}

Developmental Gain (A), Stability (B), and Loss (C)
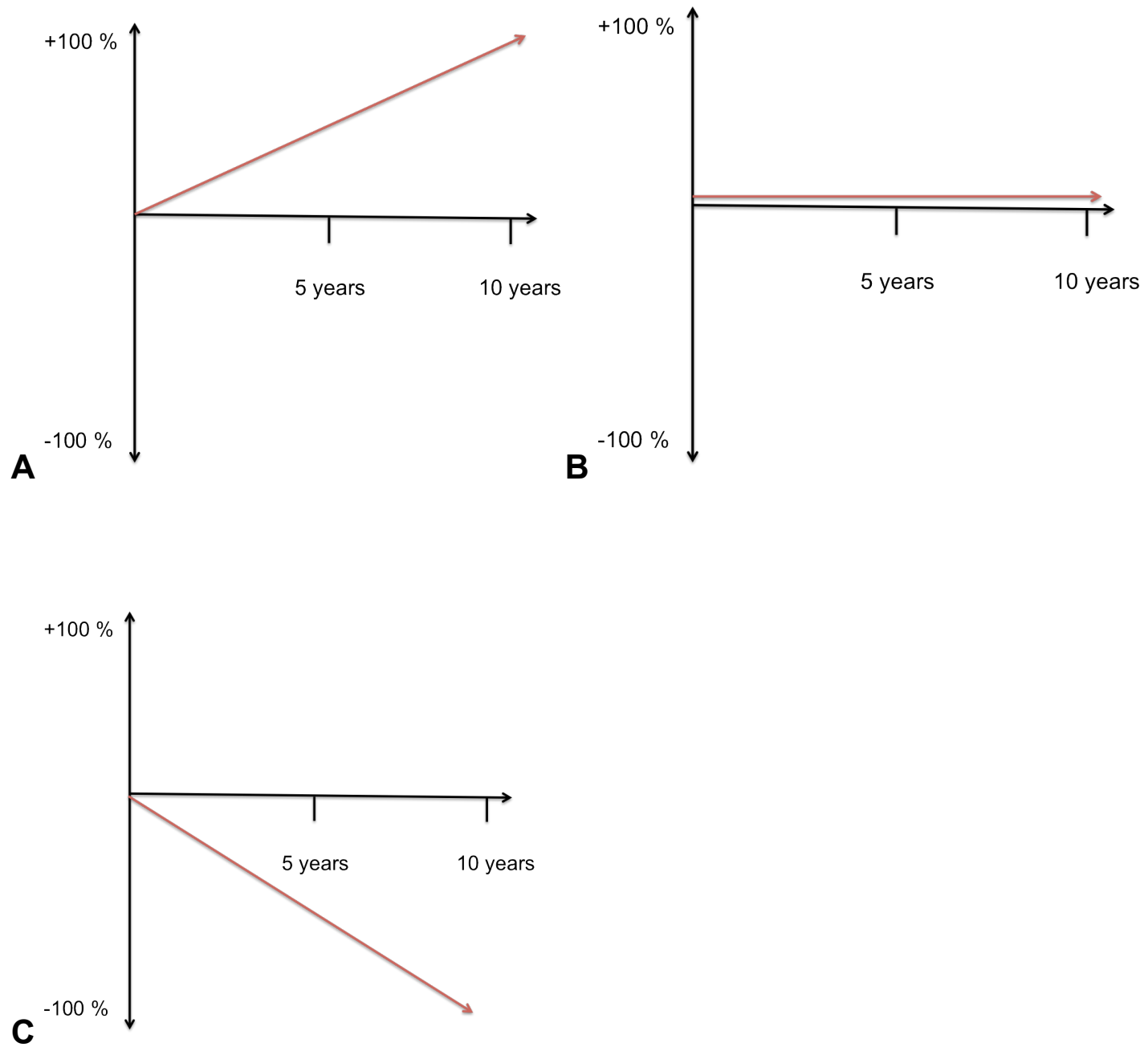


\section{Appendix B}

Procedure and Exemplary Experimental Material Used in Study 2 to Assess the Explicit (A) and Implicit Evaluation (B) of a Gain Trajectory

Using the Keys (P) Pleasant vs. (U) Unpleasant
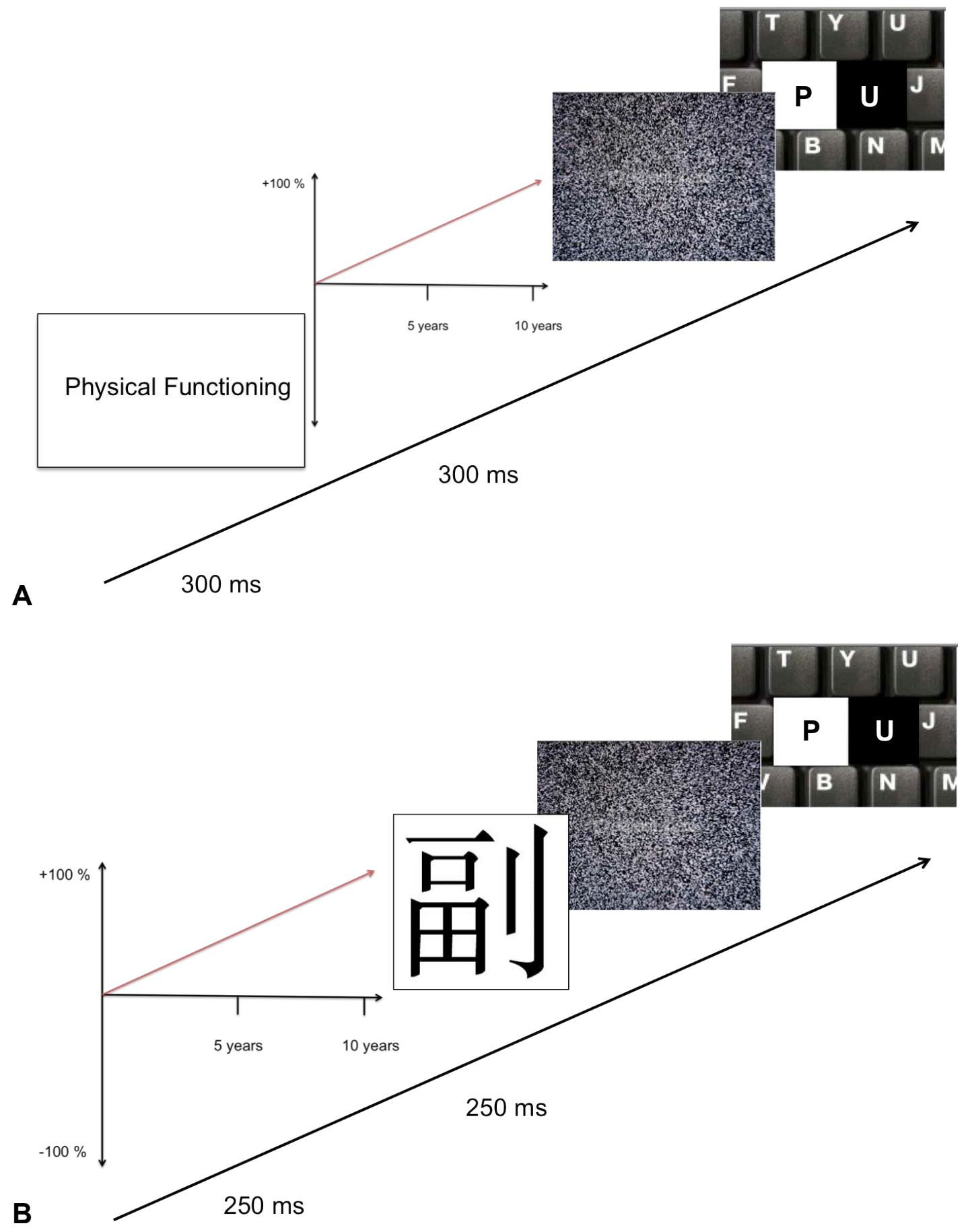


\section{Appendix C}

\section{Example of a Verbatim Manipulation of Resource Investment in Study 2}

\section{Social Relationships}

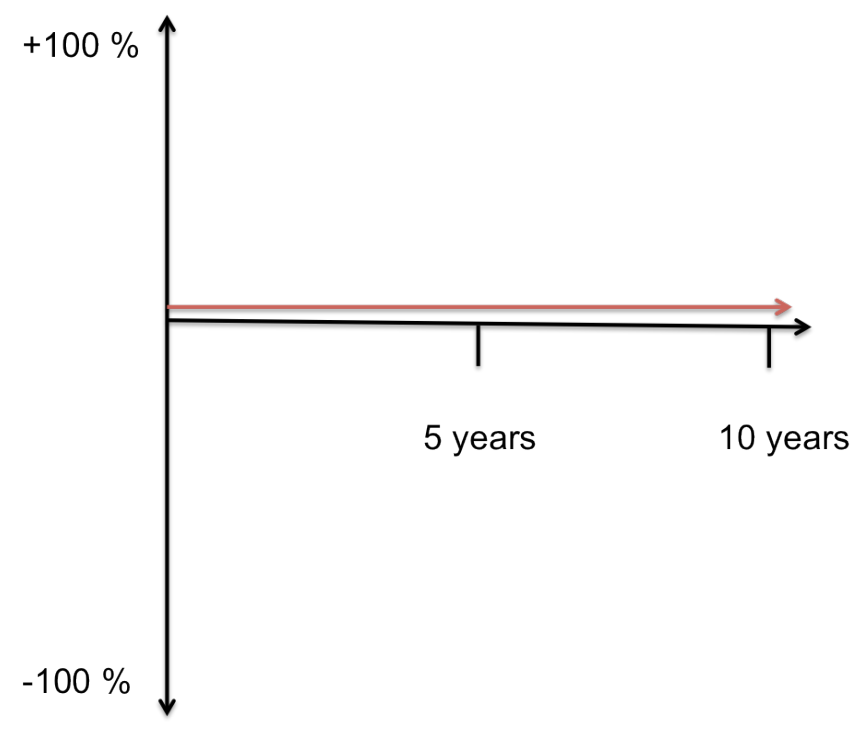

Social relationships need to be cherished. You need to maintain personal contact, you need to meet people regularly and find similar interests repeatedly. Caring for one's partner, friends, and family needs time. Taking care of social relationships is necessary to stay connected to others. This means, for example, to approach others regularly, pay attention to others and steadily be willing to compromise.

On a scale from 1 (do not agree at all) to 7 (agree completely): How would you personally evaluate your development if your social relationships were to remain stable during the next years? 